Tropical Journal of Pharmaceutical Research May 2020; 19 (5): 1045-1052

ISSN: $1596-5996$ (print); 1596-9827 (electronic)

(C) Pharmacotherapy Group, Faculty of Pharmacy, University of Benin, Benin City, 300001 Nigeria.

\title{
Molecular interaction of 4-amino-N'-(benzoyloxy)-N-(2,4- dimethylphenyl)-1,2,5-oxadiazole-3-carboximidamide with the methotrexate binding site of human DHFR, and its implication in rheumatoid arthritis
}

\author{
Shazi Shakil'1-3*, Adel M Abuzenadah ${ }^{1-3}$, Suzan M Attar ${ }^{4}$, Omar Fathaldin $^{4}$, Rajaa \\ Al-Raddadi ${ }^{5}$, Mansour I Sulaiman ${ }^{6}$ \\ ${ }^{1}$ King Fahd Medical Research Center, King Abdulaziz University, ${ }^{2}$ Department of Medical Laboratory Technology, Faculty of \\ Applied Medical Sciences, King Abdulaziz University, ${ }^{3}$ Center of Excellence in Genomic Medicine Research, King Abdulaziz \\ University, ${ }^{4}$ Rheumatology Department, King Abdulaziz University, ${ }^{5}$ Department of Community Medicine, Faculty of Medicine, \\ King Abdulaziz University, ${ }^{6}$ Department of Pharmacology, College of Medicine, King Abdulaziz University, Jeddah, Kingdom of \\ Saudi Arabia
}

*For correspondence: Email: shazibiotech@gmail.com

Sent for review: 3 February 2020

Revised accepted: 27 April 2020

\begin{abstract}
Purpose: To identify an improved lead molecule for the human dihydrofolate reductase (DHFR) inhibition that 'sits' in the same binding cavity as methotrexate by high throughput computational screening.

Methods: The 3-D structure of the DHFR binding site was examined using 'CASTp3.0'. Structure based in silico screening of about 5 million drug candidates housed in the MCULE database was performed. The obtained molecule-hits were ranked in accordance with their VINA scores, made to pass through drug-likeness filters, $\Delta G$ cut-off criterion, toxicity-checker and finally 'zero RO5 criterion'.

Results: The 'top molecule', namely, 4-amino-N'-(benzoyloxy)-N-(2,4-dimethylphenyl)-1,2,5-oxadiazole3-carboximidamide, displayed robust binding with human DHFR through 21 amino acid residues $(\Delta G=-$ $9.6 \mathrm{kcal} / \mathrm{mol}$ ) while 10 of these residues were the same as those displayed by 'methotrexate binding interactions'. It passed through relevant drug screening filters including the 'Toxicity Checker'.

Conclusion: This research work describes the molecular interaction of human DHFR with an improved lead molecule named, 4-amino-N'-(benzoyloxy)- $N$-(2,4-dimethylphenyl)-1,2,5-oxadiazole-3carboximidamide, with a $\triangle G$ of $-9.6 \mathrm{kcal} / \mathrm{mol}$, thus satisfying adequate $A D M E$ features for further in vitro and in vivo validation in the context of rheumatoid arthritis.
\end{abstract}

Keywords: Dihydrofolate reductase, In silico screening, Methotrexate, Rheumatoid arthritis, DHFR

This is an Open Access article that uses a fund-ing model which does not charge readers or their institutions for access and distributed under the terms of the Creative Commons Attribution License (http://creativecommons.org/licenses/by/4.0) and the Budapest Open Access Initiative (http://www.budapestopenaccessinitiative.org/read), which permit unrestricted use, distribution, and reproduction in any medium, provided the original work is properly credited.

Tropical Journal of Pharmaceutical Research is indexed by Science Citation Index (SciSearch), Scopus, International Pharmaceutical Abstract, Chemical Abstracts, Embase, Index Copernicus, EBSCO, African Index Medicus, JournalSeek, Journal Citation Reports/Science Edition, Directory of Open Access Journals (DOAJ), African Journal Online, Bioline International, Open-J-Gate and Pharmacy Abstracts

\section{INTRODUCTION}

Rheumatoid arthritis (RA) is an autoimmune disorder which predominantly upsets the synovial joints. It leads to disabilities and even early death events. It imposes socioeconomic burden on the ailing individuals as well as on the global community. RA is an area of continued research interest [1] and so is 'Enzoinformatics' [2]. The term 'Enzoinformatics' was coined by Shazi 
Shakil and accepted by the global scientific fraternity [2]. It is a sub-discipline of Bioinformatics that concerns enzyme-ligand binding interactions in particular $[3,4]$.

Based on the idea of 'holistic groove targeting' highlighted in our recent article [2], a putative candidate ligand was identified against the polobox-domain of PLK-1 protein. This putative drug candidate was referred to as 'SHAZ-i' [5]. Deriving inspiration from our recent and past works with reference to molecular interactions involving targets like acetylcholinesterase $[4,6]$, butyrylcholinesterase [7], Sodium-glucose cotransporter 2 [3] and matrix metalloproteinases [8]; and also varied ligands like dapagliflozin [9], lycodine [10], glimepiride [6], sotagliflozin [3] and methotrexate [11], the theme of the current article was decided.

The research was driven by the quest of identifying a promising 'seed'-skeleton which could in turn be used for design of potent dihydrofolate reductase (DHFR) inhibitors in near future. It is significant because DHFR-inhibitors have traditionally found application in the treatment of RA. Methotrexate in low dose is the drug of choice in patients with RA, because of its anti-inflammatory action through inhibition of enzymes involved in the folate pathway [12]. Furthermore, application of DHFR-inhibitors for cancer treatment has become an area of increasing research interest [13].

Hence, the objective of our research work was to identify a fresh 'seed-skeleton' for DHFRinhibition that 'sits' in the same binding cavity as methotrexate by high throughput computational screening of about 5 million drug candidates housed in the MCULE database.

\section{METHODS}

\section{CASTp3.0 protein topography probe:}

The 3-D structure of the DHFR binding site was examined using 'CASTp3.0' using the method described by Tian et al [14]. It is a free web server. The updated version of this server provides quite intuitive and comprehensive information regarding protein cavities. The protocol in turn utilizes the alpha shape method for identification of relevant protein features, for measurement of volume and area and for computing imprint [14]. The PDB ID 1 U72 was duly explored with reference to the precise site of methotrexate binding present on human DHFR using the default probe radius of $1.4 \AA$. As, this one is a complex; the drug was removed by Discovery Studio Visualizer (BIOVIA/Accelerys).
The optimized protein structure equipped for inclusion in the workflow builder was prepared using MAESTRO 9.8 [15]

\section{High throughput computational screening}

'Structure-based virtual screening' was performed in MCULE [16] whereby we screened over $5 \times 10^{6}$ putative drug candidates. The objective was to identify a fresh 'seed-skeleton' for DHFR-inhibition that 'sits' in the same binding cavity as methotrexate. A sequential input was fetched to the 'workflow builder' of the MCULE drug discovery platform. In an endeavor to provide initial search flexibility we allowed $1 \mathrm{RO} 5$ violation. A numerical value of 10 was kept for the maximum number of rotatable bonds; minimum value for $\mathrm{H}$-bond donors was entered as 1 while the sampler size was 1000 .

An input of 100 was given against 'the maximum number of most-diverse-molecules'. A value of 0.7 was assigned as the threshold similarity cutoff while the rest of the parameters remained as 'default' provided by MCULE. 'Open Babel LF' was employed for the analysis of corresponding descriptors as the screening progressed. The numerical value assigned against "the maximum number of compounds post sphere-exclusion" was $3 \times 10^{6}$ as assigned previously [4].

\section{Computational binding experiments}

The processed pdb format file (all ligands removed from the complex) of DHFR enzyme was fetched to Vina [17]. The size of the 'Grid' was assigned to be $60 \AA \times 60 \AA \times 60 \AA^{3}$ for fully covering the methotrexate binding site present on the DHFR protein. The $x, y$ and $z$ grid coordinate values which were essential to mark the 'grid-position' in the three dimensional space were obtained using the information provided with PDB ID 1U72, as done in previous studies for pertinent proteins [4]. Actually, $1 \mathrm{U} 72$ is the reality complex describing methotrexate-DHFR interaction. These values for $x, y$ and $z$ as fetched to Vina were 30.620667, 16.876455 and -1.623545 , respectively. A default Autodockprotocol was used [17], as done in previous publication [4].

\section{VINA score rankings}

The complete set of ligands obtained from the computational screening was ranked as per their VINA scores [17]. It is noteworthy that a ligand which displays a higher (negative) value as its VINA score implies a more efficient binding interaction with the active crevice of the target protein, a principle exploited by several studies 
[4,5]. Consequently, a list of 50 molecules possessing upper VINA ranks was generated.

\section{SWISS ADME profiling}

These 50 ligands were fetched to SWISS ADME to study their comparative drug profiles [18]. This included performing several kinds of tests for the ligands being investigated. Importantly, likelihood of the ligand to behave as a possible future drug molecule was predicted. This likeness to drug was measured by filters, namely Lipinsky (Pfizer), Ghose, Veber (GSK), Egan (Pharmacia) and Muegge (Bayer) filters. The ligands were also tested by 'PAINS' which is an important medicinal chemistry filter. A shortened list of candidate drugs was prepared by rejecting all the ligands that failed against more than 2 filters of drug-likeness.

\section{$\Delta \mathrm{G}$ cut off}

The obtained list of putative drug structures was further shortened by removing all the ligands that displayed a $\Delta G>-8.6 \mathrm{kcal} / \mathrm{mol}$ for their respective complexes with the human DHFR.

\section{Toxicity checker and zero RO5 violation filters}

The shortlisted set of candidate drugs was submitted to the 'Toxicity Checker' [16]. This program checks for the presence of possible moieties/sub-structures within the structure of candidate ligands that might be the hall marks of known toxic entities. The toxicity filter passed ligands were further checked for identifying the ligand(s) with zero RO5 violation.

\section{Binding interactions of the 'Top ligand' and 'methotrexate' with DHFR}

Discovery Studio 2016 (BIOVIA) was used to detach the methotrexate molecule from its complex with DHFR represented by PDB ID 1U72. Methotrexate was re-docked to the DHFR enzyme by Autodock [17]. The protocol previously published by the corresponding author of this article was also referred [19].

The grid position ( $x, y$ and $z$ co-ordinates) was defined by the numerical values as 30.620667 , 16.876455 and -1.623545 , respectively. A $60 \AA^{3}$ grid volume was used for docking. Binding interactions of the 'Top ligand' and 'methotrexate' for their respective complexes with human DHFR enzyme were studied by Discovery Studio Visualizer as well as PyMOL V1.5.0.4 and compared.

\section{RESULTS}

\section{CASTp3.0 protein topography probe outcome}

'Show Pockets' function for the protein revealed that the binding-site present on the A chain of human DHFR interacted with methotrexate through 20 amino acid residues. These residues were 17, V8, A9, L22, E30, F31, R32, F34, Q35, T56, P61, N64, L67, K68, R70, V115, Y121 and T136.

\section{High throughput computational screening output}

The screening yielded a total of 95 molecules (out of 5 million candidates) in accordance with the provided input in the workflow builder of MCULE (Figure 1).

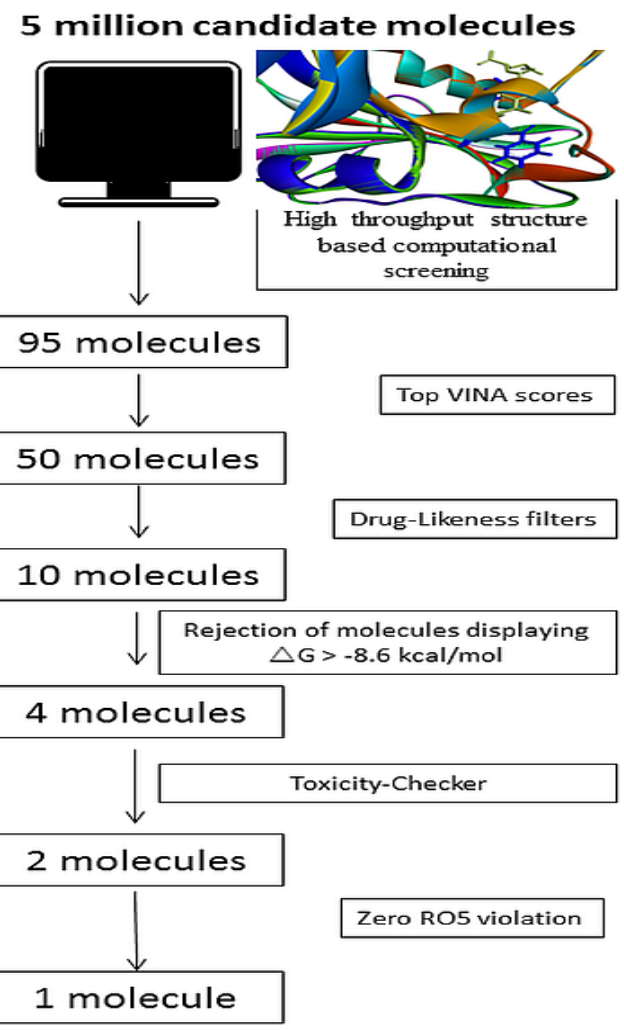

Figure 1: Sequential steps of high throughput computational screening of 5 million candidate inhibitors against human DHFR protein

Fifty molecules that displayed the upper VINA scores were chosen for further filtration. Next, these 50 ligand molecules were tested by DrugLikeness filters and also the PAINS filter by the SWISS ADME program [18]. Table 1 shows SWISS ADME profiles of the top two inhibitors obtained by screening of 5 million candidate molecules against DHFR enzyme (Table 1). 
Table 1: SWISS ADME profiles of the top two inhibitors obtained by screening 5 million candidate molecules against DHFR enzyme

\begin{tabular}{|c|c|c|}
\hline $\begin{array}{l}\text { Inhibitor name and } \\
\text { characteristics }\end{array}$ & $\begin{array}{l}\text { MCULE-5318527349-0- } \\
101\end{array}$ & $\begin{array}{l}\text { MCULE-7895890639-0- } \\
42\end{array}$ \\
\hline IUPAC Nomenclature & $\begin{array}{l}\text { 4-Amino-N'- } \\
\text { (benzoyloxy)-N-(2,4- } \\
\text { dimethylphenyl)-1,2,5- } \\
\text { oxadiazole-3- } \\
\text { carboximidamide }\end{array}$ & $\begin{array}{l}\text { 2-[(6-Bromo-4-oxo-3- } \\
\text { phenyl-3,4-dihydro-2- } \\
\text { quinazolinyl)sulfanyl]-N- } \\
\text { (2- } \\
\text { phenoxyethyl)acetamide }\end{array}$ \\
\hline Molecular formula & $\mathrm{C} 18 \mathrm{H} 17 \mathrm{~N} 5 \mathrm{O} 3$ & $\mathrm{C} 24 \mathrm{H} 20 \mathrm{BrN} 3 \mathrm{O} 3 \mathrm{~S}$ \\
\hline Molecular weight (g/mol ) & 351.36 & 510.40 \\
\hline iLOGP & 2.93 & 4.01 \\
\hline RO5 violation & 0 & 1 \\
\hline Hydrogen bond acceptors & 6 & 4 \\
\hline Hydrogen bond donors & 2 & 1 \\
\hline $\begin{array}{l}\text { Rotatable bonds } \\
\text { Toplogical polar surface } \\
\text { area (TPSA) }\left(\AA^{2}\right)\end{array}$ & $\begin{array}{l}6 \\
115.63\end{array}$ & $\begin{array}{l}9 \\
98.52\end{array}$ \\
\hline Molar refractivity & 96.91 & 130.20 \\
\hline $\begin{array}{l}\text { Gastrointestinal absorption } \\
\text { Permeability of blood brain } \\
\text { barrier }\end{array}$ & $\begin{array}{l}\text { High } \\
\text { No }\end{array}$ & $\begin{array}{l}\text { High } \\
\text { No }\end{array}$ \\
\hline Water solubility, Log S (Ali) & -5.42 & -6.89 \\
\hline $\begin{array}{l}\text { Synthetic accessibility } \\
\text { Drug-likeness and } \\
\text { medicinal chemistry } \\
\text { filters }\end{array}$ & 3.70 & 3.23 \\
\hline Lipinsky (Pfizer) & $\begin{array}{l}\text { YES } \\
\text { YES }\end{array}$ & $\begin{array}{l}\text { YES; } 1 \text { violation: } \\
\text { MW>500 } \\
\text { NO; } 2 \text { violations: }\end{array}$ \\
\hline & YES & $\begin{array}{l}M W>480, M R>130 \\
\text { YES }\end{array}$ \\
\hline Egan (Pharmacia) & YES & YES \\
\hline $\begin{array}{l}\text { Muegge (Bayer) } \\
\text { PAINS }\end{array}$ & $\begin{array}{l}\text { YES } \\
\text { YES }\end{array}$ & $\begin{array}{l}\text { NO; } 1 \text { violation: } \\
\text { XLOGP3>5 } \\
\text { YES }\end{array}$ \\
\hline
\end{tabular}

Accordingly, 10 molecules (out of 50) were selected. All the molecules that displayed a $\triangle \mathrm{G}$ value greater than $-8.6 \mathrm{kcal} / \mathrm{mol}$ were rejected. Accordingly, only 4 ligands identified as MCULE5318527349-0-101, MCULE-7895890639-0-42, MCULE-4870845335-0-48 and MCULE8593021450-0-38, remained in the active workflow. Two of these ligands i.e. MCULE4870845335-0-48 and MCULE-8593021450-0-38 failed the toxicity test. The SMILES notations of the two remaining ligands i.e. MCULE5318527349-0-101 and MCULE-7895890639-042 were fetched to CHEMSPIDER to generate their corresponding IUPAC names. Hence the respective IUPAC names for the aforementioned drug candidates i.e. MCULE-5318527349-0-101 and MCULE-7895890639-0-42 were found as 4Amino-N'-(benzoyloxy)-N-(2,4-dimethylphenyl)1,2,5-oxadiazole-3-carboximidamide and 2-[(6Bromo-4-oxo-3-phenyl-3,4-dihydro-2quinazolinyl)sulfanyl]-N-(2- phenoxyethyl)acetamide. The ligand which was a carboximidamide (MCULE-5318527349-0-101) passed through Lipinsky (Pfizer), Ghose, Veber (GSK), Egan (Pharmacia), Muegge (Bayer) and PAINS screens. However, MCULE-78958906390-42 failed the Ghose filter as it displayed 2 violations (i.e. MW > 480, MR > 130). It could not pass the Muegge (Bayer) filter as well because of 1 violation of having the value of XLOGP3 as 5.08 [Table 1]. MCULE-5318527349-0-101, the carboximidamide displayed an XLogP3 value as 3.32. Further increasing the stringency, the two aforementioned ligands were examined for having zero RO5 violations. MCULE7895890639-0-42 possessed a molecular weight of $510.40 \mathrm{~g} / \mathrm{mol}$ and hence displayed 1 RO5 violation. Also, the binding energy (negative) was found to be highest for the complex of MCULE5318527349-0-101 with DHFR $(\triangle \mathrm{G}=-9.6$ $\mathrm{kcal} / \mathrm{mol})$. Hence, MCULE-5318527349-0-101 i.e. 4-Amino-N'-(benzoyloxy)-N-(2,4-dimethylphe- 
nyl)-1,2,5-oxadiazole-3-carboximidamide was designated as the 'Top molecule' obtained after the screening in the current study.

\section{Binding interactions of the 'Top ligand' and 'methotrexate' with DHFR:}

The interacting amino acid residues for the redocked complex were observed to be the same as that of the reality crystal represented by the PDB ID 1U72. The highest (negative) $\Delta G$ value was observed for binding of MCULE5318527349-0-101 with DHFR $(\Delta G=-9.6$ $\mathrm{kcal} / \mathrm{mol})$. The runner up ligand, MCULE7895890639-0-42 displayed free energy of binding exactly equal to that of the DHFR complex involving methotrexate $(\Delta G=-8.6$ $\mathrm{kcal} / \mathrm{mol})$. The poses of these docked complexes, were studied by Discovery Studio Visualizer as well as PyMOL. Figure 2 shows the structures of the 'top molecule' and 'runner up molecule' having the IDs MCULE-5318527349-0101 and MCULE-7895890639-0-42, respectively [Figure 2]. (i) MCULE-5318527349-0-101

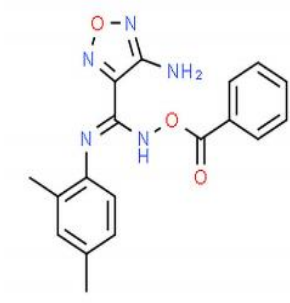

4-Amino-N'-(benzoyloxy)-N-(2,4-dimethylphenyl)1,2,5-oxadiazole-3-carboximidamide (ii) MCULE-7895890639-0-42

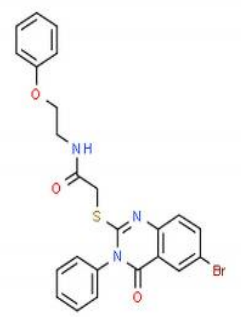

2-[(6-Bromo-4-oxo-3-phenyl-3,4-dihydrophenoxyethyl)acetamide 2-quinazolinyl)sulfanyl]-N-(2-

Figure 2: Chemical structures of the 'top molecule' [ID: MCULE-5318527349-0-101] and 'runner up molecule' [ID: MCULE-7895890639-0-42] obtained in the screening study

Figure 3 shows the '2-D-Diagram' of the 'top molecule' bound to human DHFR. Interacting amino acid residues and important interactions crucial to hold the putative inhibitor

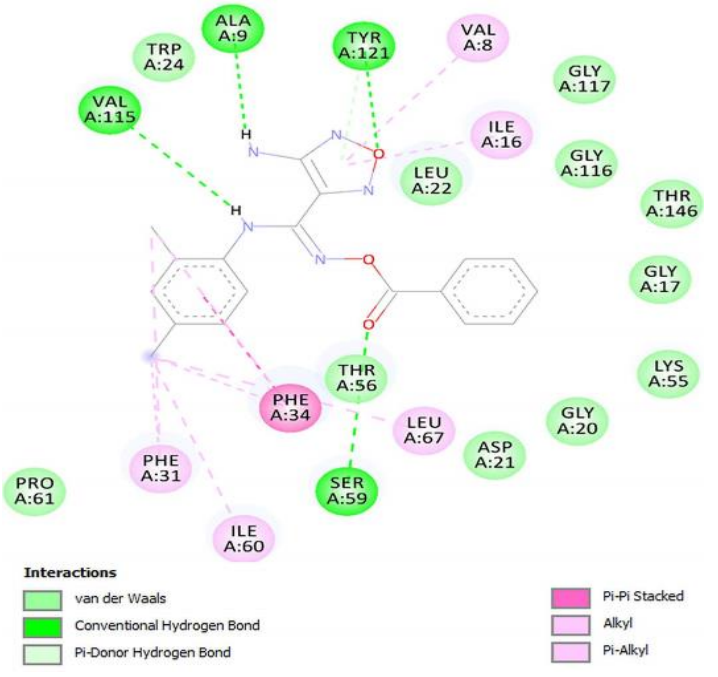

Figure 3: The '2-D-Diagram' of the 'top molecule' bound to human DHFR enzyme

within the binding cavity of the human DHFR are marked. Furthermore, we compared the binding interactions of 4-Amino-N'-(benzoyloxy)-N-(2,4dimethylphenyl)-1,2,5-oxadiazole-3-

carboximidamide with that of methotrexate with reference to their respective complexes with DHFR. The 'top molecule' interacted with DHFR through 21 amino acid residues (Table 2).

It is significant that 10 amino acid residues were found identical for the DHFR complex involving the 'top molecule' to that of the DHFRMethotrexate complex. These residues were V8, A9, L22, F31, F34, T56, P61, L67, V115 and Y121. 'Molecular Overlay' tool of Discovery Studio Visualizer was employed to generate a figure displaying methotrexate (reference ligand) as well as the 'top molecule' obtained in the current screening study simultaneously interacting with the DHFR protein. 4-Amino-N'(benzoyloxy)-N-(2,4-dimethylphenyl)-1,2,5oxadiazole-3-carboximidamide and methotrexate are represented as CPK and stick model, respectively (Figure 4 ).

Table 2: The ' $\Delta G$ ' values and important residues for the respective docked-complexes of the top 2 inhibitors and methotrexate with the DHFR enzyme

\begin{tabular}{|c|c|c|c|}
\hline $\begin{array}{l}\text { MCULE Identification } \\
\text { number/name }\end{array}$ & $\begin{array}{l}\text { MCULE- } \\
5318527349-0-101\end{array}$ & $\begin{array}{l}\text { MCULE- } \\
7895890639-0-42\end{array}$ & Methotrexate \\
\hline Free energy value $(\triangle G)$ & $-9.6 \mathrm{kcal} / \mathrm{mol}$ & $-8.6 \mathrm{kcal} / \mathrm{mol}$ & $-8.6 \mathrm{kcal} / \mathrm{mol}$ \\
\hline $\begin{array}{l}\text { 'Interacting amino acid } \\
\text { residues' for the respective } \\
\text { docked-complexes }\end{array}$ & $\begin{array}{l}\text { V8, A9, I16, G17, } \\
\text { G20, D21, L22, W24, } \\
\text { F31, F34, K55, T56, } \\
\text { S59, I60, P61, L67, } \\
\text { V115, G116, G117, } \\
\text { Y121, T146 }\end{array}$ & $\begin{array}{l}\text { V8, A9, I16, L22, } \\
\text { P26, R28, F31, F34, } \\
\text { Q35, T56, S59, I60, } \\
\text { P61, N64, L67, K68, } \\
\text { R70, Y121 }\end{array}$ & $\begin{array}{l}\text { I7, V8, A9, L22, E30, } \\
\text { F31, R32, F34, Q35, } \\
\text { T56, P61, N64, L67, } \\
\text { K68, R70, V115, } \\
\text { Y121, T136 }\end{array}$ \\
\hline
\end{tabular}




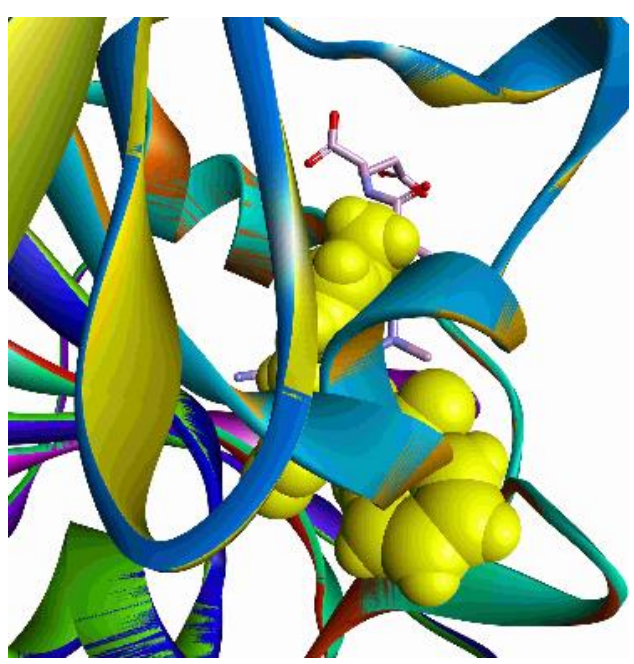

Figure 4: A close-up figure displaying methotrexate (reference ligand) as well as the 'top molecule' obtained in the current screening study simultaneously interacting with the DHFR protein for a clear comparison

\section{DISCUSSION}

The human DHFR is a known target for rheumatoid arthritis. Therefore, it is of interest to screen human DHFR for improved lead molecules in comparison with methotrexate [2022]. Such ligand(s) could either act as potent inhibitors themselves or like 'seed-structures available for highly advanced simulation studies. Top VINA scores are generally regarded as indicators of more efficient binding interactions [17].

Ligand molecules which display poor pharmacokinetic profiles tend to be rejected in the initial trail of drug-design process. For imparting initial flexibility to the screening process, at the onset we allowed all ligands that passed at least 4 out of the 6 filters mentioned in Table 1. Also, we allowed $1 \mathrm{RO} 5$ violation for the same reason [23]. A higher (negative) free energy of binding displayed by a docked complex is generally regarded as an indicator of efficient binding interactions for the corresponding protein-inhibitor pair. Therefore, the dissociation rate of such an inhibitor with its binding target would be lower and such inhibitors might be expected to possess an increased half-life [24]. Candidate molecules which possess moieties/sub-structures that might be regarded as signatures or hallmarks of known toxic entities are generally rejected in the virtual screening process. Molecule Leads are normally accepted to have an XLogP3 within 3.5.

Accordingly, MCULE-5318527349-0-101, the carboximidamide displayed an XLogP3 value of
3.32. It exhibited commendable Gl-absorption, which is considered as a positive indicator concerning putative drugs that might be given from oral route. It was found to be CNS-inactive, a good feature to avoid off-target effects. The interacting amino acid residues for the re-docked complex were observed to be the same as that of the reality crystal represented by the PDB ID 1U72. Hence, the re-docking results of methotrexate with human DHFR reinforced the accuracy of the dockings performed.

'Amidine' is a more common term used to refer to a 'carboximidamide'. In a 2011 study by Willis et al, it was observed that $\mathrm{Cl}$-amidine was able to partially inhibit arthritis in mice [25]. Rimacalib, although discontinued, was used in trials studying the treatment of Rheumatoid Arthritis and it was a 'carboximidamide' [26]. Amidine has been shown to reduce inflammation and joint destruction in arthritic mice by other authors as well [27]. Methotrexate is understood to have limitations due to toxic effects after long-term use. As DHFR-inhibitors find application in many diseases apart from arthritis, e.g. cancer, significance of novel DHFR-inhibitors or improved lead molecules displaying acceptable toxicity profiles than the contemporary drug candidates is evident.

\section{CONCLUSION}

This research work describes the molecular interaction of human DHFR with an improved lead molecule named 4-amino-N'-(benzoyloxy)$\mathrm{N}$-(2,4-dimethylphenyl)-1,2,5-oxadiazole-3carboximidamide with a $\Delta G$ of $-9.6 \mathrm{kcal} / \mathrm{mol}$ satisfying adequate ADME features for further in vitro and in vivo validation in the context of rheumatoid arthritis.

\section{DECLARATIONS}

\section{Acknowledgement}

This project was funded by National Plan for Science, Technology and Innovation (MAARIFAH) - King Abdulaziz City for Science and Technology - The Kingdom of Saudi Arabia - award no. 11-MED1553-03. The authors also acknowledge with thanks Science and Technology Unit, King Abdulaziz University for technical support.

\section{Conflict of interest}

No conflict of interest is associated with this work. 


\section{Contribution of authors}

We declare that this work was done by the authors named in this article and all liabilities pertaining to claims relating to the content of this article will be borne by the authors.

\section{Open Access}

This is an Open Access article that uses a funding model which does not charge readers or their institutions for access and distributed under the terms of the Creative Commons Attribution License (http://creativecommons.org/licenses/by/ 4.0) and the Budapest Open Access Initiative (http://www.budapestopenaccessinitiative.org/rea d), which permit unrestricted use, distribution, and reproduction in any medium, provided the original work is properly credited.

\section{REFERENCES}

1. van Vollenhoven $R$. Treat-to-target in rheumatoid arthritis - are we there yet? Nat Rev Rheumatol 2019; 15(3): 180-186.

2. Shakil S, Baig MH, Tabrez S, Rizvi SMD, Zaidi SK, Ashraf GM, Ansari SA, Khan AAP, Al-Qahtani MH, Abuzenadah AM, Chaudhary AG. Molecular and enzoinformatics perspectives of targeting Polo-like kinase 1 in cancer therapy. Semin Cancer Biol 2019; 56: 47-55.

3. Shakil S. Molecular Interaction of Anti-Diabetic Drugs With Acetylcholinesterase and Sodium Glucose CoTransporter 2. J Cell Biochem 2017; 118(11): 38553865 .

4. Shakil S. Molecular interaction of investigational ligands with human brain acetylcholinesterase. J Cell Biochem 2019; 120: 11820-11830.

5. Shakil S, Abuzinadah MF. Putative Anti-Cancer Drug Candidate Targeting the 'PLK-1-polo-box domain' by High Throughput Virtual Screening: A Computational Drug Design Study. Critical Reviews ${ }^{\mathrm{TM}}$ in Eukaryotic Gene Expression 2019; 29(3): 251-261.

6. Rizvi SM, Shaikh S, Naaz D, Shakil S Ahmad A, Haneef M, Abuzenadah AM. Kinetics and molecular docking study of an anti-diabetic drug glimepiride as acetylcholinesterase inhibitor: implication for Alzheimer's disease-diabetes dual therapy. Neurochem Res 2016; 41(6): 1475-1482.

7. Kamal MA, Shakil S, Nawaz MS, Yu QS, Holloway HW, Tan $Y, \quad Q u \quad X$, Greig NH. Inhibition of butyrylcholinesterase with fluorobenzylcymserine, an experimental Alzheimer's drug candidate: Validation of enzoinformatics results by classical and innovative enzyme kinetic analyses. CNS Neurol Disord Drug Targets 2017; 16(7): 820-827.

8. Verma A, Rizvi SM, Shaikh S, Ansari MA, Shakil S, Ghazal $F$, Siddiqui $M H$, Haneef $M$, Rehman $A$.
Compounds isolated from Ageratum houstonianum inhibit the activity of matrix metalloproteinases (MMP-2 and MMP-9): An oncoinformatics study. Pharmacogn Mag 2014; 10(37): 18-26.

9. Shaikh S, Rizvi SM, Shakil S, Riyaz S, Biswas D, Jahan $R$. Forxiga (dapagliflozin): Plausible role in the treatment of diabetes-associated neurological disorders. Biotechnol Appl Biochem 2016; 63(1): 145-150.

10. Shaikh $S$, Zainab $T$, Shakil $S$, Rizvi SM. A neuroinformatics study to compare inhibition efficiency of three natural ligands (Fawcettimine, Cernuine and Lycodine) against human brain acetylcholinesterase. Network 2015; 26(1): 25-34.

11. Shakil S, Kamal MA, Tabrez S, Abuzenadah AM, Chaudhary AG, Damanhouri GA. Molecular interaction of the antineoplastic drug, methotrexate with human brain acetylcholinesterase: a docking study. CNS Neurol Disord Drug Targets 2012; 11(2): 142-147.

12. Malik F, Ranganathan $P$. Methotrexate pharmacogenetics in rheumatoid arthritis: a status report. Pharmacogenomics 2013; 14(3): 305-314.

13. Raimondi MV, Randazzo O, La Franca M, Barone G, Vignoni E, Rossi D, Collina S. DHFR Inhibitors: Reading the Past for Discovering Novel Anticancer Agents. Molecules 2019; 24(6). pii: E1140. doi: 10.3390/molecules24061140.

14. Tian W, Chen C, Lei X, Zhao J, Liang J. CASTp 3.0: computed atlas of surface topography of proteins. Nucleic Acids Res 2018; 46(W1): W363-W367.

15. Sastry GM, Adzhigirey $M$, Day $T$, Annabhimoju $R$, Sherman $W$. Protein and ligand preparation: parameters, protocols, and influence on virtual screening enrichments. J Comput Aided Mol Des 2013; 27(3): 221-234.

16. Kiss $R$, Sandor M, Szalai FA. http://Mcule.com: a public web service for drug discovery. J Cheminform 2012; 4(Suppl 1): P17.

17. Trott O, Olson AJ. AutoDockVina: improving the speed and accuracy of docking with a new scoring function, efficient optimization, and multithreading. J Comput Chem 2010; 31(2): 455-461.

18. Daina A, Michielin O, Zoete V. SwissADME: a free web tool to evaluate pharmacokinetics, drug-likeness and medicinal chemistry friendliness of small molecules. Sci Rep 2017; 7: 42717.

19. Rizvi SM, Shakil S, Haneef M. A simple click by click protocol to perform docking: Autodock4.2 made easy for non-bioinformaticians. EXCLI J 2013; 12: 831-857.

20. Wang $N$, Ren JX, Xie Y. Identification of novel DHFR inhibitors for treatment of tuberculosis by combining virtual screening with in vitro activity assay. J Biomol Struct Dyn 2019; 37(4): 1054-1061.

21. Ruiz V, Czyzyk DJ, Valhondo $M$, Jorgensen $W L$, Anderson KS. Novel allosteric covalent inhibitors of bifunctional Cryptosporidium hominis TS-DHFR from parasitic protozoa identified by virtual screening. Bioorg Med Chem Lett 2019; 29(11): 1413-1418.

Trop J Pharm Res, May 2020; 19(5): 1051 
22. Adane L, Patel DS, Bharatam PV. Shape- and chemical feature-based 3D-pharmacophore model generation and virtual screening: identification of potential leads for $P$. falciparum DHFR enzyme inhibition. Chem Biol Drug Des 2010; 75(1): 115-126.

23. Lipinski CA. Lead- and drug-like compounds: the rule-offive revolution. Drug Discov Today Technol 2004; 1(4): 337-341.

24. Copeland RA. Conformational adaptation in drug-target interactions and residence time. Future Med Chem 2011; 3(12): 1491-1501.

25. Willis VC, Gizinski AM, Banda NK, Causey CP, Knuckley $B$, Cordova KN, Luo Y, Levitt B, Glogowska M, Chandra $P$, Kulik L, Robinson $W H$, Arend WP, Thompson $P R$,
Holers VM. $\quad \mathrm{N}$ - $\alpha$-benzoyl-N5-(2-chloro-1-iminoethyl)-Lornithine amide, a protein arginine deiminase inhibitor, reduces the severity of murine collagen-induced arthritis. J Immunol 2011; 186(7): 4396-4404.

26. Neef S, Mann C, Zwenger A, Dybkova N, Maier LS. Reduction of $S R \mathrm{Ca}^{2+}$ leak and arrhythmogenic cellular correlates by SMP-114, a novel CaMKII inhibitor with oral bioavailability. Basic Res Cardiol 2017; 112(4): 45.

27. Kawalkowska J, Quirke AM, Ghari F, Davis S, Subramanian $V$, Thompson PR, Williams RO, Fischer $R$, La Thangue NB, Venables PJ. Abrogation of collageninduced arthritis by a peptidyl arginine deiminase inhibitor is associated with modulation of T cell-mediated immune responses. Sci Rep 2016; 6: 26430. 